\title{
Du cercle fermé au réseau ouvert. Les Treize comme avant-texte de La Comédie humaine
}

\section{Thomas Conrad}

\section{(2) OpenEdition}

1 Journals

\section{Édition électronique}

URL : https://journals.openedition.org/genesis/1630

DOI : 10.4000/genesis. 1630

ISSN : 2268-1590

Éditeur :

Presses universitaires de Paris Sorbonne (PUPS), Société internationale de génétique artistique littéraire et scientifique (SIGALES)

Édition imprimée

Date de publication : 9 mai 2016

Pagination : 65-73

ISBN : 9791023105315

ISSN : $1167-5101$

\section{Référence électronique}

Thomas Conrad, « Du cercle fermé au réseau ouvert. Les Treize comme avant-texte de La Comédie humaine », Genesis [En ligne], 42 | 2016, mis en ligne le 05 juin 2017, consulté le 17 janvier 2023. URL http://journals.openedition.org/genesis/1630 ; DOI : https://doi.org/10.4000/genesis.1630 


\section{Du cercle fermé au réseau ouvert Les Treize comme avant-texte de La Comédie humaine}

Thomas Conrad

L es cycles, ces immenses assemblages de récits, se caractérisent par l'autonomie des récits qui les composent. C'est ce qui les différencie des romansfleuves et plus généralement des romans longs. Et c'est ce qui rend cet objet, malgré sa masse textuelle qui lui donne une apparence d'objectivité, particulièrement flou et mouvant. Du côté de la lecture, l'autonomie des récits permet (voire encourage) un parcours de lecture désordonné, multiple ou partiel; elle borde chaque récit d'un horizon indéfini de lectures passées, à venir, ou simplement possibles, qui en modifie le sens, la portée, ou du moins la résonance esthétique; elle permet de mettre en relation chaque récit avec tous les autres, et avec la totalité qu'ils forment entre eux, et d'infléchir ainsi son interprétation. Bref, le cycle réoriente l'attention esthétique, en la déplaçant du récit vers cet objet bien plus obscur et immatériel qu'est la relation entre les récits.

Du côté de l'écriture, la possibilité toujours ouverte d'ajouts (autographes ou allographes) modifie incessamment l'équilibre de l'ensemble et le rend particulièrement précaire. La genèse du cycle est à cet égard une genèse doublement paradoxale, par l'ambiguïté qu'y prend le statut de texte «achevé». D'une part, la genèse du cycle est une genèse ouverte : l'ajout ou le déplacement des romans, la reconfiguration du cycle, reste toujours possible, par l'écriture d'un nouvel opus ou par le jeu des rééditions. De ce fait le cycle est presque toujours un texte inachevé. D'autre part, la genèse du cycle se superpose largement au cycle lui-même. En effet, la publication de chaque roman n'est pas la fin du parcours de création, puisque chacun d'eux est aussi, en un sens, un avant-texte du cycle dans son ensemble : La Fortune des Rougon, même dans son état achevé, est encore un avant-texte du cycle des Rougon-Macquart, dont il pose de nombreux jalons. De même, Le Père Goriot (indépendamment de la question des éditions successives du texte) est à la fois un texte achevé et un avant-texte de
La Comédie humaine, puisqu'il contient le germe de récits futurs que Balzac exploitera ultérieurement ${ }^{1}$. C'est ainsi tout le cycle qui s'identifie à sa propre genèse, les avant-textes étant les éléments du texte final qu'est le cycle ${ }^{2}$ : ainsi le cycle conserve-t-il en lui-même ses stades antérieurs, comme une ville conserve, grâce à certains monuments et vestiges, une image de ce qu'elle a été auparavant.

Dès lors, il faut considérer chaque moment de l'écriture du cycle comme un équilibre plus ou moins complet, comme un palier qui a sa cohérence propre, en même temps qu'il contient des dysfonctionnements et des dynamiques qui rendent déjà possibles d'autres développements.

Un de ces paliers, un de ces équilibres instables, est la trilogie publiée par Balzac en 1833 et 1834, l'Histoire des Treize. L'Histoire des Treize, qui ouvre les Scènes de la vie parisienne, réunit trois nouvelles : Ferragus, La Duchesse de Langeais, La Fille aux yeux d'or; et une préface, sans laquelle on ne comprendrait guère ce que sont les Treize. Dès le deuxième épisode, Balzac introduit plusieurs personnages reparaissants, conformément à l'annonce de la préface. L'Histoire des Treize est donc, avant Le Père Goriot, l'un des laboratoires du retour des personnages, un des premiers cas

1. Anthony R. Pugh a mis en évidence ce processus de germination à l'œuvre dans la genèse de La Comédie humaine: Balzac trouve souvent dans un récit préalable des «germes » narratifs qui seront développés pour former la matière d'un nouveau récit : chaque roman terminé fonctionne comme un répertoire d'idées de romans (Anthony R. Pugh, Balzac's Recurring characters, Toronto, University of Toronto Press, 1974).

2. À ces avant-textes au statut ambigu, il faut bien sûr joindre les avanttextes classiques du cycle, comme le «premier plan remis à Lacroix » de Zola, ou la lettre de Balzac à Mme Hanska du 26 octobre 1834. Notons que ces avant-textes sont assez rares, car la genèse des cycles se distingue généralement assez mal de la genèse des récits qui les composent : peu d'avant-textes portent exclusivement sur le cycle, indépendamment de l'écriture de ces récits. 
où Balzac orchestre consciemment le retour des personnages en tant que tel ${ }^{3}$.

L'Histoire des Treize est à la fois une partie de la future Comédie humaine que nous connaissons, et une sorte de «protocycle», une expérimentation sur les possibilités de connexion entre récits - comme l'étaient déjà le recueil des Contes drolatiques, la nouvelle Une conversation entre onze heures et minuit, ou le projet des Études de mours au XIXe siècle. On examinera donc d'abord la cohérence propre du palier formé par l'Histoire des Treize, qui propose une des premières formules du retour des personnages. On en suivra ensuite les traces dans le reste de La Comédie humaine qui l'a englobée, de manière à renouveler l'interprétation du retour des personnages.

\section{Le cercle fermé des Treize (1833-1834)}

L'interprétation traditionnelle veut que le retour des personnages soit un des procédés par lesquels Balzac conquiert progressivement sa capacité à représenter le monde de manière réaliste. L'Histoire des Treize serait de ce point de vue un premier pas vers un réalisme plus maîtrisé, que mettra en œuvre Le Père Goriot. Bardèche estimait que «L'Histoire des Treize se passe, par nécessité, dans un milieu qui reste constamment le même et dans lequel se trouve, en fait, réalisé le principe du retour des personnages », de manière à assurer «la présence d'une société réduite qui sert de chœur ou de figuration ${ }^{4} »$. Pugh invoque lui aussi le «réalisme» pour comprendre le retour des personnages, affirmant même que «le problème esthétique soulevé par les personnages reparaissants est en réalité une subdivision du problème général du réalisme ${ }^{5}$ », l'un des buts du procédé étant de donner «une impression de véracité 6 ». Et en effet, la réapparition de personnages comme le vidame de Pamiers et la comtesse de Serizy, à l'arrière-plan de Ferragus et de La Duchesse de Langeais, n'a d'autre fonction que de mieux situer l'histoire dans le milieu aristocratique parisien.

Cette interprétation du procédé ne vaut toutefois que pour les figurants. Mais pour les Treize eux-mêmes, elle devient presque saugrenue : comment considérer que l'enjeu est de représenter les milieux sociaux parisiens, alors que les Treize sont une société secrète, qui rassemble des personnages au mépris des classes sociales, et où le forçat Ferragus côtoie le dandy de Marsay? L'unité des Treize ne vient pas d'un milieu social, mais de leur alliance secrète. Ce n'est pas par le «réalisme» qu'on expliquera que Ferragus vienne aider de Marsay (ou l'inverse)... Paradoxalement, le groupe le mieux soudé de la trilogie n'a aucune signification sociale. Autrement dit, le retour des personnages est moins un procédé réaliste qu'une contestation du réalisme.

L'Histoire des Treize correspond donc à un état spécifique du cycle, qui peut éclairer différemment l'usage du retour des personnages dans la suite de l'écriture de La Comédie humaine.

Dans sa préface, Balzac indique que «Ferragus est un premier épisode qui tient par d'invisibles liens à l' Histoire des Treize ${ }^{7}$ ». Ces «invisibles liens », les personnages qui reparaissent d'un épisode à l' autre de la trilogie, sont en effet assez discrets : contrairement à ce qu' on pourrait attendre, Balzac n'utilise jamais treize personnages reparaissants, et ne donne même pas la liste complète des «Treize». On voit certes un convoi de treize voitures dans Ferragus, et un groupe de «treize démons humains» dans La Duchesse de Langeais, mais rien n'indique une action collective des Treize en tant que groupe complet dans La Fille aux yeux d'or. Dès la publication de Ferragus dans la Revue de Paris (en avril 1833), Balzac annonçait seulement «deux autres histoires où les aventures de deux nouveaux Treize seront mises en lumière 8 », et ne comptait donc pas «compléter» sa série pour obtenir treize histoires. L'Histoire des Treize apparaît comme un cycle ouvert et partiel - à cet égard,

3. Anthony R. Pugh, ibid., p. 5-53 et p. 57-72. Pugh repère quelques personnages reparaissants au statut plus ou moins net, comme Mle de Villenoix (Louis Lambert) et le capitaine Bianchi (Les Marana), en 1832, qui s'expliqueraient plutôt par le réemploi des mêmes modèles réels pour les personnages. César Birotteau, inventé comme frère du «curé de Tours » François Birotteau, est à peu près contemporain de l'Histoire des Treize.

4. Maurice Bardèche, Une lecture de Balzac, Paris, Les Sept Couleurs, 1964 , p. 520.

5. «The aesthetic problem raised by the recurring characters is really a sub-division of the general problem of realism», Anthony R. Pugh, op. cit., p. 66.

6. «An impression of veracity» (ibid.).

7. Balzac, préface à Histoire des Treize, dans La Comédie humaine, t. V, p. 789 .

8. Balzac, postface à Ferragus, dans La Comédie humaine, ibid., p. 904. 
elle est déjà une préfiguration de La Comédie humaine tout entière, qui est faite autant de plein que de vide (sections en chantier ou quasiment vides, comme les «Scènes de la vie politique» ou les «Études analytiques », titres annoncés et jamais écrits). L'Histoire des Treize se donne d'emblée comme un état provisoire et limité du cycle, ouvert à d'autres prolongements. Cette incomplétude produit aussi un bel effet de mystère : on ne connaîtra jamais l'identité de tous les Treize, ce qui est bien le propre d'une véritable société secrète... Quel lecteur de La Comédie humaine ne serait pas tenté de mener l'enquête pour identifier les neuf membres anonymes?

Quoi qu'il en soit, si ténue que soit l'existence des Treize, elle suffit à donner à la trilogie une ossature cyclique systématique. Chacun des Treize est le protagoniste d'une nouvelle tandis qu'il joue un rôle mineur dans les deux autres. Dans Ferragus, on rencontre, autour du protagoniste éponyme, Ronquerolles (dès 1833) et de Marsay (dans la version de 1843). Dans La Duchesse de Langeais, le personnage principal, Montriveau, est lui aussi flanqué de Ronquerolles et de Marsay (à partir de la version de 1834). Enfin, dans La Fille aux yeux d'or, au second plan derrière de Marsay, c'est cette fois Ferragus qui tient compagnie à Ronquerolles. Ronquerolles, qui reparaît toujours à l'arrière-plan en tant qu' adjuvant des protagonistes, est à la fois omniprésent et inconnu : il est comme une synecdoque des Treize. Ceux-ci apparaissent aussi en personne, en tant que groupe anonyme, dans Ferragus et dans La Duchesse de Langeais : parmi eux, le lecteur doit discerner la présence incognito de Montriveau et de Ferragus, dont il sait désormais qu'ils font partie des Treize.

Pour la première fois chez Balzac, les retours de personnages forment un système. Et les Treize n'existent, littérairement parlant, que par ce système de retours de personnages, qui prend une forme rigoureuse : celle d'un cercle fermé de treize personnages (plus quelques figurants d'arrière-plan), dont chacun peut devenir tour à tour personnage principal. Si l'on peut accorder à Bardèche que «C'est probablement l'influence de Cooper qui amena Balzac à concevoir l'Histoire des Treize [...] comme une trilogie dans laquelle devait reparaître également le même groupe central», il faut noter l'originalité de Balzac par rapport à son modèle : la réapparition d'un groupe se distingue radicalement de celle d'un individu. C'est là une des originalités les plus frappantes du procédé balzacien dans La Comédie humaine : plus que par continuation chronologique (c'est-à-dire par suite ou prequel, comme chez Cooper), Balzac procède par variation actantielle (le personnage principal d'un récit devient simple figurant dans un autre, et inversement) ${ }^{9}$.

Ce système de retour des personnages s'explique par l'alliance imaginaire des Treize. Le pacte qui unit les Treize se résume en une phrase : «Je ne m'appartiens pas, je suis lié par un serment au sort de plusieurs personnes qui sont à moi comme je suis à elles ${ }^{10}$. » C'est une formule de réciprocité. Or c'est bien cette réciprocité qui fonde le retour des personnages : c'est par exemple quand les Treize sont à de Marsay, dans La Fille aux yeux d'or, qu'il est personnage principal, tandis qu'il est aux Treize dans les deux autres récits, où il devient simple figurant. En glissant d'un personnage à un autre, le cycle indique la variation des rapports de forces entre les personnages : lorsqu'un des Treize est sur le devant de la scène, c'est qu'il se subordonne les autres et que les événements sont racontés en fonction de son point de vue et de son intérêt; ailleurs, il sera figurant, car subordonné.

Le retour de personnages s'enracine ici non dans un quelconque réalisme sociologique, mais dans un imaginaire : celui du pacte et de l'alliance. On sait que les thèmes du pacte et de la société secrète frappaient profondément l'imagination de Balzac. Les Treize incarnent un idéal de concentration de l'énergie par l'association des forces individuelles : «[leur] puissance naturellement acquise peut seule expliquer certains ressorts en apparence surnaturels $11 »$. Balzac prête à cette alliance des volontés des effets qui confinent au surnaturel :

Un jour, l'un d'eux, après avoir relu Venise sauvée, après avoir admiré l'union sublime de Pierre et de Jaffier, vint à songer $[\ldots]$ aux privilèges de puissance exorbitante que ces hommes savent conquérir en confondant toutes les idées dans une seule

9. Pugh remarque ainsi que Balzac procède volontiers par amplification de l'histoire passée d'un personnage; mais il analyse cette démarche comme celle qui consiste à écrire des « suites rétrospectives» (des prequels, dirait-on, ou dans les termes de Genette, des suites analeptiques). Or l'originalité de Balzac n'est pas là, et notamment par rapport à Fenimore Cooper : elle est dans le fait que c'est presque toujours un personnage secondaire dont le passé est développé.

10. Balzac, La Fille aux yeux d'or, dans La Comédie humaine, op. cit., t. V, p. 1099.

11. Balzac, préface à Histoire des Treize, dans ibid., p. 789. 
volonté. [...] Il présuma que la société devait appartenir tout entière à des gens distingués qui, à leur esprit naturel, à leurs lumières acquises, à leur fortune, joindraient un fanatisme assez chaud pour fondre en un seul jet ces différentes forces. Dès lors, immense d'action et d'intensité, leur puissance occulte, contre laquelle l'ordre social serait sans défense, y renverserait les obstacles, foudroierait les volontés, et donnerait à chacun d'eux le pouvoir diabolique de tous 12 .

Cette souveraineté secrète a des résonances nettement politiques. La pensée politique de Balzac est tout entière dans cette idée de concentration du pouvoir : despotisme, droit d'aînesse, admiration pour les jésuites, etc. «Ce qui représente pour lui l'idéal politique, c'est, écrit Curtius, la concentration de l'énergie motrice ${ }^{13}{ }$, contre l'individualisme qui disperse les énergies individuelles. Aussi le pacte qui donne aux Treize leur souveraineté n'est-il pas sans rappeler la formule du contrat social de Rousseau :

Or comme les hommes ne peuvent engendrer de nouvelles forces, mais seulement unir et diriger celles qui existent, ils n'ont plus d'autre moyen pour se conserver, que de former par aggrégation une somme de forces qui puisse l'emporter sur la résistance, de les mettre en jeu par un seul mobile et de les faire agir de concert.

Cette somme de forces ne peut naître que du concours de plusieurs : mais la force et la liberté de chaque homme étant les premiers instrumens de sa conservation, comment les engagera-t-il sans se nuire, et sans négliger les soins qu'il se doit ? [...] Tel est le problème fondamental dont le contract social donne la solution.

[...] Si donc on écarte du pacte social ce qui n'est pas de son essence, on trouvera qu'il se réduit aux termes suivans. Chacun de nous met en commun sa personne et toute sa puissance sous la suprême direction de la volonté générale; et nous recevons en corps chaque membre comme partie indivisible du tout ${ }^{14}$.

On reconnaît l'égalité des participants, le vocabulaire énergétique (mouvement, résistance, forces), et le thème de la volonté générale dans laquelle se fondent les volontés particulières, en constituant la souveraineté. Les Treize aussi acquièrent une souveraineté : ils sont «treize rois inconnus, mais réellement rois 15 »; Ferragus est « roi légitime» des Dévorants, et son nom est en fait un nom dynastique, Ferragus XXIII. De Marsay tient «un sceptre plus puissant que ne l'est celui des rois modernes $16 »$. On comprend alors que Balzac attribue au pacte une puissance presque surnaturelle, puisqu'il institue une dimension nouvelle qui dépasse l'individu. C'est en ce sens qu'Alain peut dire que Ronquerolles, représentant des Treize, est «comme un dieu17» : dans la métaphysique de Balzac, l'énergie et la volonté ne sont-elles pas la substance même du monde?

Mais, bien sûr, le pacte des Treize est un contrat social restreint (une élite de treize personnes) et inversé (un contrat «antisocial», dirigé contre la médiocrité ambiante), qui institue «[un] monde à part dans le monde, hostile au monde18 » : c'est le cas de dire qu'ils font «concurrence à l'État-Civil»... Dans l'imaginaire de la société secrète, le thème du contrat est ambivalent : d'un côté, il est l'expression la plus aboutie de l'individualisme que la Révolution a hérité des Lumières; de l'autre, il inverse le contrat social en le restreignant à une élite héroïque et en le dirigeant contre la médiocrité bourgeoise issue de cette même Révolution. Le retour des personnages, dans l'Histoire des Treize, est intimement lié à cette concentration des volontés qui retourne le contrat social contre lui-même en fondant une nouvelle aristocratie.

\section{Ouvrir le cercle}

Que reste-t-il de cette dimension dans La Comédie humaine? Apparemment, peu de chose. Dès l'Histoire des Treize, d'ailleurs, le bilan est ambigu : après tout, les Treize échouent. Chantal Massol montre bien que la trilogie raconte trois défaites : les Treize, vaincus, ne parviennent pas à régénérer le monde postrévolutionnaire. Dès 1833 , Balzac affirmait dans sa préface que la royauté des Treize cesse à la mort de Napoléon : «le pouvoir de la société secrète, au moment où le romancier entend faire apparaître

\section{Ibid., p. 791.}

13. Ernst Robert Curtius, Balzac, Paris, Grasset, 1933, p. 232.

14. Jean-Jacques Rousseau, Du contract social, dans Euvres complètes, dir. Bernard Gagnebin et Marcel Raymond, Paris, Gallimard, 1964, p. 360-361 [je souligne].

15. Balzac, préface à Histoire des Treize, op. cit., p. 792.

16. Balzac, La Fille aux yeux d'or, op. cit., p. 1 084-1 085.

17. Alain, Balzac, Paris, Gallimard, 1999, p. 65.

18. Balzac, préface à Histoire des Treize, op. cit., p. 791. 
son existence, n'est plus [...]. Les Treize sont donc anachroniques ${ }^{19}$ ». Les «invisibles liens » de la société secrète se dissolvent quand s'installe la société bourgeoise de la Restauration et de la monarchie de Juillet. Autrement dit, le modèle du cercle fermé des Treize est abandonné aussitôt qu'il est conçu, et ne représente qu'un équilibre local et provisoire dans la genèse de La Comédie humaine.

Il est vrai que La Comédie humaine n' est pas une simple amplification de l'Histoire des Treize. Mais on peut repérer certaines continuités, en particulier le thème de la société secrète, dont le principe «aristocratique» (car élitaire) s'accommode très bien des transpositions à d'autres classes et à d'autres objectifs. L'une de ces sociétés secrètes est signalée dans Gobseck, en 1835. L'usurier parle de son appartenance à un groupe de collègues qui s'échangent les informations financières :

Liés par le même intérêt, nous nous rassemblons à certains jours de la semaine au café Thémis, près du Pont-Neuf. Là, nous nous révélons les mystères de la finance. Aucune fortune ne peut nous mentir, nous possédons les secrets de toutes les familles. [...] Celui-ci surveille la masse judiciaire, celui-là la masse financière; l'un la masse administrative, l'autre la masse commerciale. [...] Comme moi, tous mes confrères ont joui de tout, se sont rassasiés de tout, et sont arrivés à n'aimer le pouvoir et l'argent que pour le pouvoir et l'argent même20.

À première vue, ce groupe d'usuriers, de «capitalistes », n'a guère de points communs avec les Treize : cette société secrète est profondément bourgeoise, ancrée dans les mécanismes sociaux et financiers de la société postrévolutionnaire. Pourtant, Gobseck retrouve pour la décrire les accents de l'Histoire des Treize : «Le Pouvoir et le Plaisir ne résument-ils pas tout votre ordre social? Nous sommes dans Paris une dizaine ainsi, tous rois silencieux et inconnus, les arbitres de vos destinées ${ }^{21}$.»

Reste-t-il un lien entre ce pacte et un système de retours des personnages, comme c'était le cas avec les décentrements complémentaires des Treize? Avons-nous dans La Comédie humaine une «histoire des usuriers » unie par les «liens invisibles» de leur association, comme nous avions une «histoire des Treize» ? Contre toute attente, oui : les ressorts secrets de cette association réapparaissent en 1844 dans Les Employés, à propos de la lutte entre Baudoyer et Rabourdin pour obtenir une place de chef de division dans l'administration. La femme de Baudoyer demande l'aide de son oncle, l'usurier Bidault (dit Gigonnet) : elle apprend que «passé cette heure, on ne peut le trouver qu'à un café nommé café Thémis, un singulier nom... [...] on dit qu'il y joue tous les soirs aux dominos avec son ami monsieur Gobseck22». La société secrète des usuriers (Mitral, Bidault, et finalement Gobseck) entre alors en action et triomphe. C'est la grande différence avec les Treize : les usuriers, eux, n'échouent pas.

Une autre association secrète, qui commande elle aussi un cycle dans La Comédie humaine, est celle qui se construit autour de Vautrin. Le pacte entre Vautrin et ses protégés est une nouvelle version du pacte des Treize. On retrouve dans la bouche de Vautrin les références à Venise sauvée et au Contrat social de Rousseau, dont Vautrin «[se] glorifie d'être l'élève 23 ». Le pacte entre Lucien et Vautrin-Herrera, où « chacun est tour à tour dominateur et dominé 24 », reprend bien celui des Treize. Sa connotation diabolique le rend néanmoins asymétrique : c'est un «pacte d'homme à démon, d'enfant à diplomate ${ }^{25}$ ». La formule du pacte n'est alors plus la réciprocité mais la subordination : un pacte «d'homme à démon » n'est pas la même chose qu'un pacte qui donne à chacun «le pouvoir diabolique de tous ${ }^{26}$ ». Vautrin déclare à Lucien : «vous m'appartenez comme la créature est au créateur 27 ». Ce rapport de subordination se répercute dans le type de retour de personnages qui organise le «cycle de Vautrin », où Vautrin a manifestement une position centrale et dominante : il devient «une espèce de colonne vertébrale qui, par son horrible influence, relie pour ainsi dire Le Père

19. Chantal Massol, Une poétique de l'énigme. Le récit herméneutique balzacien, Genève, Droz, 2006, p. 302 (et plus généralement p. 297-304). 20. Balzac, Gobseck, dans La Comédie humaine, op. cit., t. II, p. 976-977.

21. Ibid. Une «trentaine» dans Le Papa Gobseck en 1835.

22. Balzac, Les Employés, dans La Comédie humaine, op. cit., t. VII, p. 1033-1034.

23. Balzac, Le Père Goriot, dans La Comédie humaine, op. cit., t. III, p. 220 .

24. Balzac, Splendeurs et misères des courtisanes, dans La Comédie humaine, op. cit., t. VI, p. 510 .

25. Balzac, Illusions perdues, dans La Comédie humaine, op. cit., t. V, p. 703 .

26. Balzac, préface à Histoire des Treize, op. cit., p. 791.

27. Balzac, Illusions perdues, op. cit., p. 703. 
Goriot à Illusions perdues, et Illusions perdues à [Splendeurs et misères des courtisanes] ${ }^{28}$ ». Le destin de Vautrin, rallié finalement à la monarchie de Juillet, est semblable à celui des Treize.

Ils sont paisiblement rangés sous le joug des lois civiles, de même que Morgan, l'Achille des pirates, se fit, de ravageur, colon tranquille, et disposa sans remords, à la lueur du foyer domestique, de millions ramassés dans le sang, à la rouge clarté des incendies 29 .

Les Treize commencent comme des Achille et finissent comme des rentiers. Fin de l'épopée? Peut-être. À moins qu'il n'y ait une continuité entre l'époque où l'on amasse des trésors «dans le sang» et celle où on en dispose «sans remords »... Comme Vautrin, les Treize survivent peut-être, transformés, sous la monarchie de Juillet. C'est bien ce que Balzac suggère à la fin du Contrat de mariage. De Marsay explique, dans une lettre à son ami Paul de Manerville, sa participation à la vie politique de la monarchie de Juillet, aux côtés de « Ronquerolles, Montriveau, les Grandlieu, La Roche-Hugon, Serizy, Féraud et Granville ${ }^{30} »$. Le lecteur aura remarqué les deux premiers noms de la liste... Certains des Treize sont donc encore associés. Est-ce un ralliement à la «médiocratie» ambiante ? Non, si l'on en juge par leur orientation politique, typiquement balzacienne (absolutisme, machiavélisme, opposition au royalisme sentimental) :

Nous sommes capables de tout pour le bonheur du pays et pour le nôtre. Les questions personnelles en fait de roi sont aujourd'hui des sottises sentimentales [...]. La politique n'est plus là, mon cher. Elle est dans l'impulsion à donner à la nation en créant une oligarchie où demeure une pensée fixe de gouvernement [...], au lieu de laisser tirailler le pays en mille sens différents, comme nous l'avons été depuis quarante ans dans cette belle France [...] à laquelle il faudrait un système plutôt que des hommes ${ }^{31}$.

C'est la continuation des Treize par d'autres moyens. Il s'agit toujours de concentrer le pouvoir dans un réseau d'alliances, en assumant cette fois ouvertement son sens politique :

Mon vrai père fait partie du ministère anglais. Nous aurons des intelligences en Espagne par les Évangélista [...]. Montriveau, mon cher, est lieutenant-général; il sera certes un jour ministre de la guerre, car son éloquence lui donne un grand ascendant sur la chambre. Voici Ronquerolles ministre d'état et du conseil privé. Martial de La Roche-Hugon est ambassadeur, il nous apporte en dot le maréchal duc de Carigliano et tout le croupion de l'empire qui s'est soudé si bêtement à l'échine de la restauration. Serizy mène le conseil-d'état où il est indispensable. Grandville tient la magistrature à laquelle appartiennent ses deux fils; les Grandlieu sont admirablement bien en cour; Féraud est l'âme de la coterie Gondreville, bas intrigants qui sont toujours en haut, je ne sais pourquoi. Appuyés ainsi, qu'avons-nous à craindre? Nous avons un pied dans toutes les capitales, un œil dans tous les cabinets, et nous enveloppons l'administration sans qu'elle s'en doute ${ }^{32}$.

De Marsay évoque même les Treize à mots couverts :

Nous nous sommes amusés, quelques bons vivants et moi, comme des mousquetaires noirs, gris et rouges, pendant douze années, ne nous refusant rien, pas même une entreprise de flibustier par ci par là ; maintenant nous allons nous mettre à secouer les prunes mûres dans l'âge où l'expérience a doré les moissons ${ }^{33}$.

L'alliance politique esquissée dans Le Contrat de mariage se distingue cependant des Treize, en ce qu'elle ne constitue pas un cercle fermé. Les retours de personnages ont alors pris leur forme balzacienne définitive, et s'étendent en ramifications indéfinies : Serizy, Grandlieu, Gondreville, tissent des liens vers d'autres romans et d'autres réseaux, qui à leur tour, etc. De proche en proche, c'est toute la société bourgeoise qui est structurée par ces réseaux d'alliances. Et l'on sait combien Balzac a pris soin de cartographier ces alliances : complots politiques, mariages de raison (et bien souvent mésalliances, qui allient noblesse et argent), liens commerciaux et financiers, etc.

Et dans une autre Scène de la vie politique, commencée en 1839 et restée inachevée, Le Député d'Arcis, on retrouve encore les Treize, avec qui Balzac semble n'en avoir jamais complètement fini : on apprend à propos de Maxime de

\footnotetext{
28. Balzac, Splendeurs et misères des courtisanes, op. cit., p. 851.

29. Balzac, préface à Histoire des Treize, op. cit., p. 787.

30. Balzac, Le Contrat de mariage, dans La Comédie humaine, op. cit., t. III, p. 646-647.

31. Ibid., p. 647.

32. Ibid., p. 651-652.

33. Ibid., p. 652.
} 
Trailles que, dans sa jeunesse, il avait fait partie «d'une association commencée dans un but de plaisir, d'amusement (Voir LES TREIZE), et qui tourna naturellement à la politique cinq ans avant la révolution de Juillet ${ }^{34}$ ».

Balzac décide ainsi tardivement que Maxime de Trailles aura été un des Treize. C'est donc que Balzac, depuis la préface de l'Histoire des Treize, a changé d'avis sur leur dissolution. Au lieu de se disperser à la mort de Napoléon (en 1821), les Treize ont seulement évolué vers la politique (en 1825 - Le Contrat de mariage datait les projets politiques de De Marsay en 1827). Ce flottement chronologique révèle la difficulté de la transition entre deux moments : d'un côté, la constitution d'une société secrète aristocratique en révolte contre la société bourgeoise; de l'autre, l'insertion de cette contre-société dans la société bourgeoise - ou si l'on veut la représentation de cette société bourgeoise comme entremêlement de réseaux d'alliances, au fond indiscernable des sociétés secrètes. Il semble en tout cas acquis, pour Balzac, que l'alliance des Treize, comme l'«horrible influence» de Vautrin, survit en s'intégrant en profondeur à la monarchie de Juillet.

Le Député d'Arcis précise le sens politique des alliances, par la bouche de Rastignac, qui, devenu ministre, se met à disserter sur la politique en véritable disciple de De Marsay. Il en vient tout naturellement au thème de la volonté qui «est tout dans la politique » :

Quand on est tout, comme fut Napoléon, il vient un moment où il faut se faire suppléer; et comme on a écarté les gens supérieurs, le grand tout ne trouve pas de suppléant. Le suppléant, c'est ce qu'on nomme un cabinet, et il n'y a pas de cabinet en France 35 .

L'unité despotique d'un seul (Napoléon) n'est plus à l'ordre du jour. L'unité ne sera pas assurée par un seul, mais par une souveraineté collective : c'est le rôle du cabinet, du gouvernement, considéré comme une volonté collective. Les Treize étaient le paradigme de cette unité collective : on comprend alors qu'ils réapparaissent, métamorphosés en hommes politiques. Une telle souveraineté collective peut alors prétendre au titre de «suppléant» du «grand tout» (Napoléon ou le Peuple). Autrement dit, la totalité n'est pas le fait d'une unification mais d'une alliance. Cette conclusion n'est pas que politique, et concerne tout aussi bien la totalité cyclique qu'est La Comédie humaine.
On a vu qu'une spécificité du cycle, du point de vue de la genèse, est que ses éléments peuvent être considérés comme son «avant-texte» : en ce sens, l'Histoire des Treize est à la fois une préfiguration et une partie de La Comédie humaine; à la fois un petit cycle autonome, où Balzac expérimente le retour des personnages, et un des éléments englobés dans le vaste cycle de La Comédie humaine.

L'évolution qu'on a suivie ici, des Treize originels aux dernières œuvres de Balzac (La Dernière incarnation de Vautrin en 1847, Le Député d'Arcis commencé en 1839, mais resté inachevé), permet de saisir un des chemins suivis par la genèse de La Comédie humaine, sur un de ses points cruciaux : le caractère systématique du retour des personnages balzaciens.

Or, de ce point de vue, les Treize sont une nouveauté importante dans la genèse du cycle. Balzac n'y adopte pas la formule de la suite (où un personnage principal reparaît de récit en récit, chronologiquement) : modèle nettement héroïque, où le personnage principal récurrent est mis à part des personnages ordinaires. Il ne s'agit pas non plus de reprendre un personnage pour en faire l' «incarnation d'une idée » (comme Louis Lambert) ${ }^{36}$ ou de faire revenir des personnages secondaires communs pour donner une «impression de véracité » ponctuelle (comme dans Même histoire $)^{37}$. Dès la préface des Treize, publiée le 10 mars 1833 dans la Revue de Paris, Balzac constitue un système de personnages reparaissants, ce qui rompt avec l'idée du retour des personnages comme répétition et reprise, au profit d'une logique de permutations et d'alternances.

C'est encore un modèle «aristocratique» du cycle, où les personnages reparaissants sont un cercle fermé de personnages privilégiés, qui forme une alternative à la société bourgeoise dans laquelle il s'inscrit. Ces thèmes et ces personnages chemineront de Gobseck (1835) jusqu'à La Dernière Incarnation de Vautrin (1847), en passant par Le Contrat de mariage (1835) et Le Député d'Arcis (1839) : autant de traces d'une des transformations et des

34. Balzac, Le Député d'Arcis, dans La Comédie humaine, op. cit., t. VIII, p. 805 .

35. Ibid., p. 810-811.

36. «embodiment of a key idea» (Pugh, op. cit., p. 71).

37. «impression of veracity» (ibid., p. 66). 
réinterprétations qui affectent cette forme-sens, sans jamais en effacer complètement l'origine. Progressivement, l'opposition entre le pacte de la société secrète et les alliances de la société bourgeoise, entre l'envers et l'endroit de l'histoire contemporaine, finira par s'estomper : La Comédie humaine élargit sans limite le procédé, faisant virtuellement de chaque figurant le héros d'un roman à venir, selon un modèle pour ainsi dire «démocratique» du cycle. Au cours de la genèse du cycle, les Treize eux-mêmes s'intègrent de plus en plus profondément dans la société issue de 1830, tout comme l'Histoire des Treize est absorbée dans la structure plus vaste de La Comédie humaine qui se présente finalement comme une immense cartographie des réseaux d'alliances de la société contemporaine.

Thomas CONRAD est maître de conférences à l'ENS (Paris). Ses travaux portent sur les cycles romanesques (Poétique des cycles romanesques. De Balzac à Volodine, à paraître). 


\section{Du cercle fermé au réseau ouvert}

La genèse du cycle se confond largement avec le cycle lui-même : chaque élément d'un cycle en est aussi un avant-texte. Considéré par rapport à La Comédie humaine que nous connaissons, l'Histoire des Treize est un de ces avant-textes, d'autant plus intéressant qu'il forme, dès 1833 , un ensemble autonome de récits interconnectés, c'est-à-dire un premier modèle de cycle, une première expérimentation sur le retour systématique des personnages. Or il est manifeste que l'unité des Treize n'est pas de nature sociale : le retour de personnages s'enracine, mais dans l'imaginaire politique du pacte social. L'unité de l'œuvre balzacienne est ainsi liée à un idéal de souveraineté politique collective, dont le modèle, loin de disparaître entièrement dans La Comédie humaine telle qu'elle se développera ensuite dans les années 1830 et 1840, reste souterrainement actif jusque dans les dernières œuvres du cycle. Le cercle fermé des Treize, sans disparaître, devient un réseau ouvert. Les Treize se fondent dans la société bourgeoise sans perdre leur identité, tout comme l'Histoire des Treize s'insère finalement dans La Comédie humaine, avant-texte ineffaçable dans le texte qu'il préfigure.

The cycle's genesis is an integral part of the cycle itself: each element of a cycle is also its "avant-texte". Considered in relation to La Comédie Humaine such as we know it today, the Histoire des Treize is one of these "avant-textes" all the more interesting that it forms, as early as 1833, an autonomous set of interconnected stories, that is, a first model of a cycle, a first experiment with the systematic return of certain characters. It is clear that the unity of the "Thirteen" is not social: the characters' return takes root but in the political imagination of the social pact. The unity of Balzac's works is thus linked to the ideal of a collective political supremacy whose model, far from disappearing in the Comédie Humaine such as it developed/ evolved in the 1830s and 1840s, remained covertly active up to the last works of the cycle. The closed circle of the "Thirteen" did not disappear, but became an open network. The "Thirteen" are absorbed in the bourgeois society without losing their identity, just as the Histoire des Treize finally finds its place in the Comédie Humaine, an indelible "avant-text" in the text it heralds.

Die Genese eines Zyklus deckt sich größten Teils mit dem Zyklus selbst. Jedes Element eines Zyklus ist gleichzeitig auch ein Avanttexte. Im Verhältnis zur Comédie humaine, wie wir sie kennen, ist die Histoire des Treize einer dieser Avant-Textes und er ist umso interessanter, als er, seit 1833, ein autonomes Ensemble von miteinander verbundenen Erzählungen, d.h. ein erstes Modell des Zyklus, ein erstes Experimentieren mit der systematischen Wiederkehr von Personen ist. Nun ist offensichtlich, dass die Einheit der Treize nicht sozialer Natur ist: die Wiederkehr der Personen fasst Fuß, jedoch in der politischen Vorstellungswelt des Gesellschaftsvertrags. Die Einheit des Balzacschen Werks ist so an ein Ideal kollektiver politischer Souveränität geknüpft, dessen Modell - weit entfernt davon, in der Comédie humaine, wie sie sich in den 1830er- und 1840er-Jahren entwickelt, ganz zu verschwinden - bis in die letzten Werke des Zyklus unterschwellig wirksam bleibt. Der geschlossene Kreis der Treize wird, ohne zu verschwinden, ein offenes Netzwerk. Die Treize ordnen sich in die bürgerliche Gesellschaft ein, ohne ihre Identität zu verlieren, und die Histoire des Treize wird schließlich Teil der Comédie humaine, ein unauslöschlicher Avant-Texte des Werkes, das er ankündigt.
La génesis de los ciclos se confunde ampliamente con los ciclos mismos: cada elemento de un ciclo constituye al mismo tiempo un pre-texto. La Historia de los Trece, considerada en relación con La comedia humana que conocemos, es uno de esos pre-textos, en este caso particularmente interesante ya que se conforma, desde 1833, como un conjunto autónomo de relatos interconectados, es decir, un primer modelo de ciclo, una primera experimentación de la recurrencia sistemática de los personajes. Ahora bien, es evidente que la unidad de Los Trece no es de naturaleza social: es en el imaginario político del pacto social donde esta unidad se consolida. La unidad de la obra de Balzac está vinculada, de este modo, con un ideal de soberanía política colectiva, cuyo modelo no desaparece completamente en La comedia humana, tal como se desarrollará luego, en los años 1830 y 1840, sino que, por el contrario, permanece subterráneamente activo hasta en las últimas obras del ciclo. El ciclo cerrado de los Trece, sin desaparecer, se convierte en una red abierta. Los Trece se funden en la sociedad burguesa sin perder su identidad, del mismo modo que la Historia de los Trece se inserta finalmente en La comedia humana, pre-texto imborrable en el texto que prefigura.

A génese do ciclo largamente se confunde com o ciclo em si mesmo: cada elemento de um ciclo é também um seu antetexto. Comparada com La Comédie humaine que conhecemos, a Histoire des Treize é um desses antetextos, especialmente interessante porque forma, já em 1833, um conjunto independente de histórias interligadas, ou seja, um primeiro modelo de ciclo, uma primeira experimentação sobre o sistemático retorno das personagens. No entanto, é manifesto que a unidade dos Treze não é social na sua natureza: o retorno de personagens entronca sim no imaginário político do pacto social. A unidade da obra balzaquiana liga-se a um ideal de soberania política coletiva, cujo modelo, longe de desaparecer inteiramente em La Comédie humaine tal como ela se desenvolverá nas décadas de 1830 e 1840 , se mantém subterraneamente ativo até às últimas obras do ciclo. O círculo fechado dos Treze, em vez de desaparecer, torna-se uma rede aberta. Os Treze baseiam-se na sociedade burguesa sem perderem a identidade; do mesmo modo, assim a Histoire des Treize acaba por se encaixar em La Comédie humaine, antetexto indelével no texto que antecipa.

La genesi del ciclo si confonde in buona parte con il ciclo stesso: ogni elemento di un ciclo ne è anche un avantesto. Considerata rispetto alla Commedia umana, la Storia dei Tredici è uno di questi avantesti, tanto più interessante in quanto dal 1833 , forma un insieme autonomo di racconti interconnessi, ossia un primo modello di ciclo, una prima sperimentazione sul ritorno sistematico dei personaggi. È però chiaro che l'unità dei Tredici non è di natura sociale: il ritorno dei personaggi si radica, ma nell'immaginario politico del patto sociale. L'unità dell'opera balzacchiana si lega così a un ideale della sovranità politica collettiva, il cui modello, lungi dallo scomparire interamente nella Commedia umana così come si svilupperà successivamente negli anni 1830 e 1840, resta sotterraneamente attivo fin nelle ultime opere del ciclo. Il circolo chiuso dei Tredici non scompare ma diventa un sistema aperto. I Tredici si dissolvono nella società borghese senza perdere la loro identità, proprio come la Storia dei Tredici s'inserisce alla fine nella Commedia umana, avantesto incancellabile nel testo che prefigura. 